\title{
Ipsilateral Combined Monteggia Type II Equivalent and Galeazzi Injuries with Radial Head Fracture in an Adult Patient - Case Report and Literature Review
}

\author{
Silvan Azzopardi, Lyndon W. Mason*, Abijit Guha and Andrew Rogers
}

Department of Trauma and Orthopaedic, Royal Gwent Hospital, Cardiff Road, Newport, NP20 2UB, UK

\begin{abstract}
We report the occurrence of a radial head fracture (Mason Johnston type 4), combined with a Monteggia type II equivalent fracture and Galeazzi fracture pattern in the same forearm of a 19-year-old man involved in a road traffic accident.

Fractures of the radius and ulna bones were openly reduced and fixed; the radial head fracture was managed conservatively. The case was complicated by elbow dislocation and radioulnar instability, which was treated conservatively with a satisfactory outcome.
\end{abstract}

\section{Case History}

A 19-year-old right hand dominant building engineer was brought to our accident and emergency department following a road traffic collision. He was road testing a motorcycle when he lost control. The motorcycle was travelling at a low speed. His right knee contacted the ground and the motorbike landed on his right thigh. At the same time his left arm struck a stationary car.

On admission, the patient was alert, orientated and haemodynamically stable. On secondary survey and radiographic examination the patient was found to have a left sided posterior elbow dislocation accompanying a radial head fracture (Mason Johnston type 4$)[1,2]$. The radial head was also dislocated posteriorly, with a concomitant posterior angulation of the proximal ulnar diaphyseal fracture (Monteggia fracture Bado classification type II equivalent) [3] (Figure 1and2). In the same forearm there was a fracture of the distal third of radial shaft and dislocation of the distal radio-ulnar joint (Galeazzi fracture) [4] (Figure 3and4). Other injuries included a closed; transverse comminuted mid-shaft fracture right femur, a Gustilo and Anderson grade II [5] open stellate fracture of the right patella.

This gentleman was otherwise fit and well with a no significant medical history, not on any medications and reported no drug allergies. The only past surgical history was an undisplaced fracture at the neck of left radius sustained four and a half years previously. This old fracture was managed with a sling and healed with a full recovery of range of movements. Initial management was instituted in the emergency

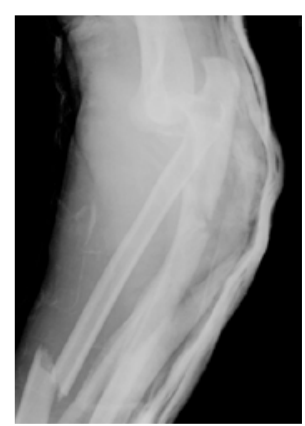

Figure 1: Illustrates a lateral radiograph of the proximal forearm and elbow. Fracture to the distal radial metadiaphysis and proximal ulnar metadyaphysis are apparent, along with elbow dislocation and radial head fracture. department following the usual advanced trauma life support protocol. All fractures were temporarily splinted. Urgent debridement of patellar fracture and operative stabilization of left upper limb were performed on the night of admission, and internal fixation of the stellate patellar fracture and femoral fracture were performed on the following day's trauma list.

Open reduction and internal fixation of the left upper limb was undertaken through Henry's approach to the radius and a dorsal approach to the ulna. The distal radius was reduced and plated with a 7-hole dynamic compression plate (Synthes, Glutz Bltzheim-Str.1-3, and 4500 Solothurn, Switzerland) maintaining the radial bow. The proximal ulna fracture was reduced and held with an 8-hole dynamic compression plate. On regaining both the length of the radius and ulna, both the elbow and distal radioulnar joint appeared relocated. The radial

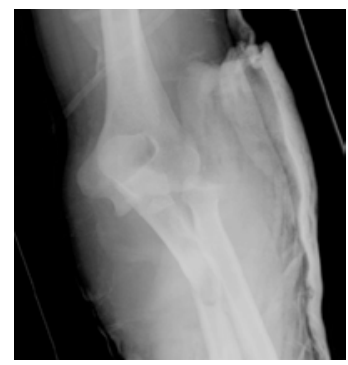

Figure 2: Illustrates an anteroposterior radiograph of the elbow. Fracture to the ulnar proximal metadyaphysis and radial head combined with an elbow dislocation is pictured.

*Corresponding author: Lyndon W. Mason, Department of Trauma and Orthopaedic, Royal Gwent Hospital, Cardiff Road, Newport, NP20 2UB, UK, Tel:+4407947038874 E-mail: mrlyndonmason@me.com

Received March 27, 2012; Accepted April 19, 2012; Published April 23, 2012

Citation: Azzopardi S, Mason LW, Guha A, Rogers A (2012) Ipsilateral Combined Monteggia Type II Equivalent and Galeazzi Injuries with Radial Head Fracture in an Adult Patient - Case Report and Literature Review. J Trauma Treat 1:130. doi:10.4172/2167-1222.1000130

Copyright: (c) 2012 Azzopardi S, et al. This is an open-access article distributed under the terms of the Creative Commons Attribution License, which permits unrestricted use, distribution, and reproduction in any medium, provided the original author and source are credited. 
Citation: Azzopardi S, Mason LW, Guha A, Rogers A (2012) Ipsilateral Combined Monteggia Type II Equivalent and Galeazzi Injuries with Radial Head Fracture in an Adult Patient - Case Report and Literature Review. J Trauma Treat 1:130. doi:10.4172/2167-1222.1000130

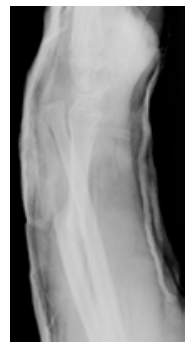

Figure 3: Show a lateral and anteroposterior radiographs, illustrating a volar angulated radial distal metadyaphyseal fracture with a dorsal ulnar head dislocation.

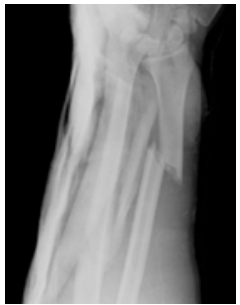

Figure 4: Show a lateral and anteroposterior radiographs, illustrating a vola angulated radial distal metadyaphyseal fracture with a dorsal ulnar head dislocation.

head fracture was managed conservatively as it was in good alignment. At the same time, the right patellar fracture was irrigated and debrided. On the next day's trauma list, the femoral fracture was addressed with a 400 mm T2 nail (Stryker UK Limited, Stryker House, Hambridge Road, Newbury, and Berkshire). The stellate patellar fracture was reduced and held with cerclage wire and crossed Kirschner wires. The wound was closed primarily.

Post operatively the left elbow remained posterolaterally unstable, dislocating at 45 degrees. Reduction was held in deep flexion, greater than 90 degrees, for the first week in a plaster cast (Figure 5). The plaster cast was replaced after 6 days reducing the flexion. At two weeks, radiographic examination revealed a reduced elbow and wrist with maintenance of fracture reduction. A hinged brace was provided and gentle extension commenced within a $45^{\circ}$ angle. On a two week follow up, extension was found to be possible to $20^{\circ}$ and the patient was advised to increase the extension to full within seven days. Throughout this time supination was a problem, falling $30^{\circ}$ short of the contralateral side. No pain was experienced at the wrist; however, the ulnar appeared to dorsally sublux in pronation. A subsequent MRI confirmed ruptured ulnar attachments of the triangular-fibro cartilage complex associated with ulnar styloid fracture. The patient was offered the option of triangular-fibro cartilage complex stabilization, however he was happy with his progress. On further monthly follow up appointments, progress was noted in both the forearm strength and range of movement and no further intervention was advocated. On his last clinic review, he had regained a full range of motion in both his elbow and wrist, and was not complaining of any pain. His final radiographs are seen in Figure 6and7.

\section{Discussion}

Occurring alone Monteggia and Galeazzi fracture patterns are not uncommon, occurring in 1-2\% [6] and 3\% [7] of forearm fractures respectively. Their combinations in an ipsilateral forearm, both pure
Monteggia/Galeazzi fractures and their equivalents have been reported but rarely [6,8-16]. The combination of a Monteggia fracture pattern and radial head fracture is also reported but again is rare [17-20]. Bado also described Monteggia type II equivalent fracture pattern, which involve posterior dislocation of the elbow with radial neck fractures [3]. To our knowledge a radial head fracture (Mason Johnston type 4) [1,2], combined with a Monteggia type II equivalent fracture [3] and Galeazzi fracture pattern [4] has previously not been described.

The mechanism of injury described previously for ipsilateral simultaneous Monteggia and Galeazzi fractures involve the axial loading of a hyperpronated and extended forearm [9,14,21,22]. However, Reckling et. al. [23] described Monteggia equivalent type II fractures occurring with direct trauma to a supinated forearm. Wilkins describes that in Galeazzi fractures, dorsal type dislocation occurs with a pronated forearm and palmer dislocation with a supinated forearm [24]. We believe in our case, a complicated mechanism occurred where the forearm was axially loaded initially in a pronated position, however the forearm was forcibly supinated on impact with the stationary car causing the Monteggia type II equivalent fracture. The radial head fracture, likely occurred on dislocation.

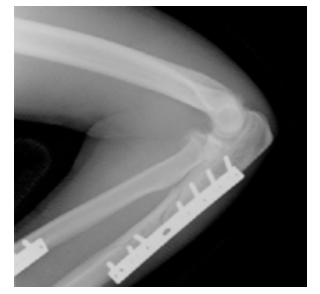

Figure 5: Lateral radiograph of the left elbow taken 3 days postoperative The elbow is kept in joint in polymer cast obtaining greater than 90 degrees of flexion. The plate and screw fixation of both the ulnar and radius is noted.

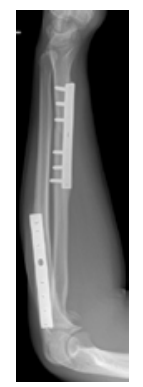

Figure 6: Final lateral and anteroposterior radiographs of the left forearm, taken 6 months post injury. Both the elbow and wrist joints are intact.

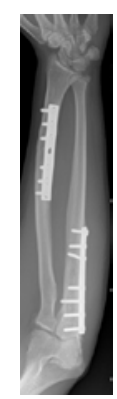

Figure 7: Final lateral and anteroposterior radiographs of the left forearm, taken 6 months post injury. Both the elbow and wrist joints are intact. 
Citation: Azzopardi S, Mason LW, Guha A, Rogers A (2012) Ipsilateral Combined Monteggia Type II Equivalent and Galeazzi Injuries with Radial Head Fracture in an Adult Patient - Case Report and Literature Review. J Trauma Treat 1:130. doi:10.4172/2167-1222.1000130

The management of Monteggia and Galeazzi fractures on their own is complicated, Reckling reporting approximately $80 \%$ of adults having less than optimum results in regards to Monteggia fractures but satisfactory results in Galeazzi fracture treatment [23]. Monteggia fractures in children can generally be treated closed except in cases where the ulnar cannot be reduced [25-27] or where there is fracture separation of the proximal radial epiphysis [16]. Reckling reported results in children were invariably good [23]. Nevertheless, some believe open reduction internal fixation result in superior results [28]. It is generally accepted that Monteggia fractures in adults are treated by open reduction and internal fixation.

Campbell termed the Galeazzi fracture as a "fracture of necessity", as it necessitates surgical intervention [29]. The deforming forces acting on the distal radius, i.e. the weight of the hand, pronator quadrates, brachioradilis and the thumb abductors, all act to make this fracture pattern highly unstable. Hughston [30] reported unsatisfactory outcomes in 35 (92\%) out of 38 cases treated in plaster cast. Reckling reported good outcomes in patients treated with open reduction and plate fixation, followed by plaster immobilization in full Supination [23]. He felt that a major factor in the stability of the Galeazzi fracture was the disruption of the distal radioulnar joint, a notion seconded by Mikic [31]. Complete dislocation of the distal radio-ulnar joint always involves rupture of the triangular-fibro cartilage disc and of the associated dorsal and volar distal radio-ulnar ligaments. Reckling felt that maintenance of supination approximated these injuries and allowed them to heal.

In our case, the initial problem was the elbow instability. In retrospect the comminuted and shortened radial head fracture was likely the major contributor to the instability. Mohanial et al. [18] reported an excellent clinical outcome following radial head fixation in such a case. We believe that radial head excision would have been disastrous due to the likely interosseous membrane injury [32]. Conservative management was sufficient to regain stability in our case. The distal radio ulnar joint initially appeared stable and we felt that regaining range of movement at the elbow was priority. Such a combined injury would be difficult to treat with above elbow immobilization for 8 weeks as recommended by Reckling [23] as the elbow stiffness resulting would be a major concern. At this later stage we have an option to fix the ulnar styloid [31] and arthroscopically address the triangular fibro cartilage complex and associated ligaments. However, the patient was happy with his outcome and refused any further surgical intervention. He has since returned to full activity.

\section{Conclusion}

In conclusion, we report the occurrence of a radial head fracture (Mason Johnston type 4), combined with a Monteggia type II equivalent fracture and Galeazzi fracture pattern in the same forearm with elbow dislocation. This is the first reported case of this combination of injury. The patient underwent open reduction and internal fixation, but was complicated by continued elbow and distal radioulnar instability. This proximal ad distal instability was treated conservatively with a satisfactory outcome.

\section{References}

1. Johnston GW (1962) A follow-up of one hundred cases of fracture of the head of the radius with a review of the literature. Ulster Med J 31: 51-56.

2. Mason ML (1954) Some observations on fractures of the head of the radius with a review of one hundred cases. Br J Surg 42: 123-132.

3. Bado JL (1967) The Monteggia lesion. Clin Orthop Relat Res 50: 71-86.
4. Galeazzi R (1934) Di una particolare sindrome traumatica dello scheletro dell'avambraccio. Soc Loath chir 2: 12.

5. Gustilo RB, Anderson JT (1976) Prevention of infection in the treatment of one thousand and twenty-five open fractures of long bones: retrospective and prospective analyses. J Bone Joint Surg Am 58: 453-458.

6. Clare DJ, Corley FG, Wirth MA (2002) Ipsilateral combination Monteggia and Galeazzi injuries in an adult patient: a case report. J Orthop Trauma 16: 130134

7. Wong PC (1967) Galeazzi fracture--dislocations in Singapore 1960-64 incidence and results of treatment. Singapore Med J 8: 186-193.

8. http://www.ispub.com/journal/the-internet-journal-of-third-world-medicine/ volume-1-number-2/combined-monteggia-and-galeazzi-fractures-in-a-child-sforearm.html

9. Kristiansen B, Eriksen AF (1986) Simultaneous type II Monteggia lesion and fracture-separation of the lower radial epiphysis. Injury 17: 51-52.

10. Maeda H, Yoshida K, Doi R, Omori O (2003) Combined Monteggia and Galeazzi fractures in a child: a case report and review of the literature. J Orthop Trauma 17: 128-131.

11. Mann CJ, Jeer P, Housden P, Hyde ID (2000) Combined Galeazzi and Monteggia forearm fracture. J R Soc Med 93: 144-145.

12. Rappold G (1994) [Combination of Monteggia and Galeazzi injury of the same arm]. Unfallchirurgie 20: 111-114.

13. Rodgers WB, Smith BG (1993) A type IV Monteggia injury with a dista diaphyseal radius fracture in a child. J Orthop Trauma 7: 84-86.

14. Shonnard PY, DeCoster TA (1994) Combined Monteggia and Galeazz fractures in a child's forearm. A case report. Orthop Rev 23: 755-759.

15. http://www.ispub.com/journal/the-internet-journal-of-orthopedic-surgery/ volume-1-number-2/type-iii-monteggia-injury-with-ipsilateral-distal-radius-andulna-fracture.html

16. Song KS, Bae KC (2004) Type III Monteggia Equivalent Fracture with Ipsilateral Distal Radial Epiphyseal and Ulnar Metaphyseal Fracture in a Child: Case Report. J Korean Orthop Assoc 39: 563-565.

17. Jupiter JB, Leibovic SJ, Ribbans W, Wilk RM (1991) The posterior Monteggia lesion. J Orthop Trauma 5: 395-402.

18. Mohanial P, Morgan G, Nathan S (2005) Monteggia fracture dislocation with radial head fracture: The need for early recognition and review of the literature. Injury Extra 36: 550-552.

19. Preston CF, Chen AL, Wolinsky PR, Tejwani NC (2003) Posterior dislocation of the elbow with concomitant fracture of the proximal ulnar diaphysis and radia head: a complex variant of the posterior monteggia lesion. J Orthop Trauma 17: $530-533$

20. Ring D, Jupiter JB, Simpson NS (1998) Monteggia fractures in adults. J Bone Joint Surg Am 80: 1733-1744.

21. Evans EM (1949) Pronation injuries of the forearm, with special reference to the anterior Monteggia fracture. J Bone Joint Surg Br 31B: 578-88.

22. Sood A, Khan O, Bagga T (2008) Simultaneous monteggia type I fracture equivalent with ipsilateral fracture of the distal radius and ulna in a child: a case report. J Med Case Reports 2: 190

23. Reckling FW (1982) Unstable fracture-dislocations of the forearm (Monteggia and Galeazzi lesions). J Bone Joint Surg Am 64: 857-863.

24. Wilkins KE, O'Brien E (1996) Fractures of the distal radius and ulna. In Fractures in Children. CAJ Rockwood, Lippincott-Raven, KE Wilkins, Philadelphia, USA.

25. Frazier JL, Buschmann WR, Insler HP (1991) Monteggia type I equivalent lesion: diaphyseal ulna and proximal radius fracture with a posterior elbow dislocation in a child. J Orthop Trauma 5: 373-375

26. Ovesen O, Brok KE, Arreskøv J, Bellstrøm T (1990) Monteggia lesions in children and adults: an analysis of etiology and long-term results of treatment. Orthopedics 13: 529-534.

27. Walsh HP, McLaren CA, Owen R (1987) Galeazzi fractures in children. J Bone Joint Surg Br 69: 730-733.

28. Osada D, Tamai K, Kuramochi T, Saotome K (2001) Three epiphyseal fractures 
Citation: Azzopardi S, Mason LW, Guha A, Rogers A (2012) Ipsilateral Combined Monteggia Type II Equivalent and Galeazzi Injuries with Radial Head Fracture in an Adult Patient - Case Report and Literature Review. J Trauma Treat 1:130. doi:10.4172/2167-1222.1000130

Page 4 of 4

(distal radius and ulna and proximal radius) and a diaphyseal ulnar fracture in a seven-year-old child's forearm. J Orthop Trauma 15: 375-377.

29. http://jama.ama-assn.org/content/162/1/79.1.short

30. Hughston JC (1957) Fracture of the distal radial shaft; mistakes in management. J Bone Joint Surg Am 39-A: 249-264.
31. Mikic ZD (1975) Galeazzi fracture-dislocations. J Bone Joint Surg Am 57: 10711080.

32. Edwards GS Jr, Jupiter JB (1988) Radial head fractures with acute distal radioulnar dislocation. Essex-Lopresti revisited. Clin Orthop Relat Res: 61-69. 